\title{
Construction and Analysis of Projected Deformed Products
}

\author{
Raman Sanyal • Günter M. Ziegler
}

Dedicated to Victor Klee.

Received: 8 July 2008 / Revised: 27 January 2009 / Accepted: 30 January 2009 /

Published online: 4 March 2009

(C) Springer Science+Business Media, LLC 2009

\begin{abstract}
We introduce a deformed product construction for simple polytopes in terms of lower-triangular block matrix representations. We further show how Gale duality can be employed for the construction and the analysis of deformed products such that specified faces (e.g., all the $k$-faces) are "strictly preserved" under projection.
\end{abstract}

Thus, starting from an arbitrary neighborly simplicial ( $d-2)$-polytope $Q$ on $n-1$ vertices, we construct a deformed $n$-cube, whose projection to the last $d$ coordinates yields a neighborly cubical d-polytope. As an extension of the cubical case, we construct matrix representations of deformed products of (even) polygons (DPPs) which have a projection to $d$-space that retains the complete $\left(\left\lfloor\frac{d}{2}\right\rfloor-1\right)$-skeleton.

In both cases the combinatorial structure of the images under projection is determined by the neighborly simplicial polytope $Q$ : Our analysis provides explicit combinatorial descriptions. This yields a multitude of combinatorially different neighborly cubical polytopes and DPPs.

As a special case, we obtain simplified descriptions of the neighborly cubical polytopes of Joswig and Ziegler (Discrete Comput. Geom. 24:325-344, 2000) as well as of the projected deformed products of polygons announced by Ziegler (Electron. Res. Announc. Am. Math. Soc. 10:122-134, 2004), a family of 4-polytopes whose "fatness" gets arbitrarily close to 9 .

\footnotetext{
R. Sanyal

Department of Mathematics, UC Berkeley, Berkeley, CA 94720-3840, USA

e-mail: sanyal@math.berkeley.edu

G.M. Ziegler $(\bowtie)$

Inst. Mathematics, MA 6-2, TU Berlin, 10623 Berlin, Germany

e-mail: ziegler@math.tu-berlin.de
} 
Keywords Polytope projections - Deformed projected products $\cdot$ Combinatorial description of polytopes $\cdot$ Block matrices $\cdot$ Gale duality $\cdot$ Neighborly cubical polytopes

\section{Introduction}

Some remarkable geometric effects can be achieved for projections of "suitablydeformed" high-dimensional simple polytopes. This includes the Klee-Minty cubes [8], the Goldfarb cubes [4], and many other exponential examples for variants of the simplex algorithm, but also the "neighborly cubical polytopes" first constructed by Joswig and Ziegler [7]. A geometric framework for "deformed product" constructions was provided by Amenta and Ziegler [1].

Here we introduce a generalized deformed products construction. In terms of this construction, the previous version by Amenta and Ziegler concerned deformed products of rank 1. The new construction is presented in matrix version (that is, as an $\mathcal{H}$-polytope). Iterated deformed products are thus given by lower-triangular block matrices, where the blocks below the diagonal do not influence the combinatorics of the product (for suitable right-hand sides).

The deformed products $P$ are constructed in order to provide interesting images after an affine projection $\pi: P \rightarrow \pi(P)$. The deformations we are after are designed so that certain classes of faces of the deformed product $P$, e.g., all the $k$-faces, are "preserved" by a projection to some low-dimensional space, i.e., mapped to faces of $\pi(P)$. In the combinatorially convenient situation, the faces in question are strictly preserved by the projection; we give a linear algebra condition that characterizes the faces that are strictly preserved (Projection Lemma 2.5). We also identify a situation where all nontrivial faces of $\pi(P)$ arise as images $\pi(F)$ of faces $F \subset P$ that are strictly preserved (Corollary 2.8).

The conditions dictated by the Projection Lemma may be translated via a nonstandard application of Gale duality [5, Sect. 6.3], [16, Sect. 6] into conditions about the combinatorics of an auxiliary polytope $Q$.

As an instance of this set-up, we show how neighborly cubical d-polytopes arise from projections of a deformed $n$-cube where all the $\left(\left\lfloor\frac{d}{2}\right\rfloor-1\right)$-faces are preserved by the projection. The precise form of the matrix representation of the $n$-cube and the combinatorics of the resulting polytopes are dictated via Gale duality by a neighborly simplicial (!) $(d-2)$-polytope with $n-1$ vertices. As special cases, we obtain the neighborly cubical polytopes first obtained by Joswig and Ziegler [7] and also geometric realizations for neighborly cubical spheres as described by Joswig and Rörig [6] and originally by Babson, Billera, and Chan [2].

Finally, we construct and analyze projected deformed products of (even) polygons (PDPP polytopes) as the images of a deformed product of $r$ even polygons projected to $\mathbb{R}^{d}$. The projection is designed to strictly preserve all the $\left(\left\lfloor\frac{d}{2}\right\rfloor-1\right)$-faces (and additional $\frac{d}{2}$-faces if $d$ is even). This produces in particular the two-parameter family of four-dimensional polytopes from [18], for which the "fatness" parameter introduced in [17] gets as large as $9-\varepsilon$. We present a new construction (drastically simplified and systematized) and a complete combinatorial description of these polytopes. 
This work is based on the Diploma thesis [13]; see also the research announcements in [18] and [19]. The "wedge product" polytopes of Rörig and Ziegler [12] provide another interesting instance of "deformed high-dimensional simple polytopes." A further analysis shows that the neighborly cubical polytopes, the PDPP polytopes, and the wedge products do exhibit a wealth of interesting polyhedral surfaces, including the "surfaces of unusually high genus" by McMullen, Schulz, and Wills [10], and equivelar surfaces of type $(p, 2 q)$. Topological obstructions that prevent a suitable projection of "deformed products of odd polygons," or of the wedge product polytopes, will be presented by Rörig and Sanyal [11].

\section{Basics}

In this section we recall basic properties and notation about the main objects of this paper, convex polytopes. Readers new to the country of polytopia will find useful information in the well-known travel guides $[5,16]$, while the frequent visitors might wish to skim the section for possibly nonstandard notation.

One of the main messages this article tries to convey is that it pays off to work with polytopes in explicit coordinates (matrix representation). Classically, there are two fundamental ways of viewing a polytope in coordinates: the interior or $\mathcal{V}$-representation, and the exterior or $\mathcal{H}$-representation. For $\mathcal{V}$-polytopes "with few vertices," Perles [5, Chap. 6] had developed Gale duality as a powerful tool. In this article, we will apply Gale duality for the analysis of projected simple $\mathcal{H}$-polytopes. The basics for this will be developed in this section.

\subsection{Polytopes in Coordinates}

For the rest of the section, let $P \subset \mathbb{R}^{d}$ be a full-dimensional polytope. In its interior or $\mathcal{V}$-presentation, $P=\operatorname{conv} V$ is given as the convex hull of a finite point set $V=$ $\left\{v_{1}, \ldots, v_{m}\right\} \subset \mathbb{R}^{d}$, and $V$ is inclusion-minimal with respect to this property. The elements of $V$ are called vertices, with notation vert $P=V$. For a nonempty subset $I \subseteq[m]=\{1, \ldots, m\}$, the set $V_{I}=\left\{v_{i}: i \in I\right\}$ forms a face of $P$ if there is a linear functional $\ell: \mathbb{R}^{d} \rightarrow \mathbb{R}$ such that $\ell$ attains its maximum over $P$ on $F=$ conv $V^{\prime}$. The $\operatorname{dimension} \operatorname{dim} F$ is the dimension of its affine span. The empty set is also a face of $P$ of dimension -1 . The collection $\mathcal{F} P$ of all faces of $P$, ordered by inclusion, is a graded, atomic, and coatomic lattice with dim +1 as its rank function. We denote by $\mathcal{F} \partial P:=\mathcal{F} P \backslash\{P\}$ the face poset of the boundary of $P$. We say that two polytopes are of the same combinatorial type if their face lattices are isomorphic as abstract posets. A polytope $P$ is simplicial if small perturbations applied to the vertices do not alter its combinatorial type. Equivalently, every $k$-face of $P(k<\operatorname{dim} P)$ is the convex hull of exactly $k+1$ vertices. The quotient $P / F$ of $P$ by a face $F$ is a polytope with face lattice isomorphic to $\mathcal{F} P_{\geq F}=\{G \in \mathcal{F} P: F \subseteq G\}$. If $F=\{v\}$ is a vertex, then $P / v$ is called a vertex figure at $v$. 
The polytope $P$ is given in its exterior or $\mathcal{H}$-presentation if $P$ is the intersection of finitely many halfspaces. That is, if there are (outer) normals $a_{1}, \ldots, a_{n} \in \mathbb{R}^{d}$ and displacements $b_{1}, \ldots, b_{n} \in \mathbb{R}$ such that

$$
P=\bigcap_{i=1}^{n}\left\{x \in \mathbb{R}^{d}: a_{i}^{\top} x \leq b_{i}\right\},
$$

where we assume that the collection of normals is irredundant, thus discarding any one of the halfspaces changes the polytope. The hyperplanes $H_{i}=$ $\left\{x \in \mathbb{R}^{d}: a_{i}^{\top} x=b_{i}\right\}$ are said to be facet defining; the corresponding $(d-1)$-faces $F_{i}=P \cap H_{i}$ are called facets. More compactly, we think of the normals $a_{i}$ as the rows of a matrix $A \in \mathbb{R}^{n \times d}$ and, with $b \in \mathbb{R}^{n}$ accordingly, write

$$
P=P(A, b)=\left\{x \in \mathbb{R}^{d}: A x \leq b\right\} .
$$

For any subset $F \subseteq P$, let eq $F=\left\{i \in[n]: F \subset H_{i}\right\} \subseteq[n]$ be its equality set. Clearly, $F \subseteq \bigcap_{i \in \text { eq } F} F_{i}$; in case of equality, the set $F$ is a face of $P$. Denote by $A_{I}$ the submatrix of $A$ induced by the row indices in $I \subseteq[n]$. Thus any face $F$ is given by $F=P \cap\left\{x: A_{I} x=b_{I}\right\}$ for $I=$ eq $F$. The collection of equality sets of faces ordered by reverse inclusion is isomorphic to $\mathcal{F} P$. The polytope $P$ is simple if its combinatorial type is stable under small perturbations applied to the bounding hyperplanes. Equivalently, every nonempty face $F$ is contained in no more than $\mid$ eq $F \mid=d-\operatorname{dim} F$ facets.

\subsection{Gale Duality}

Let $P \subset \mathbb{R}^{d}$ be a $d$-polytope, and let the rows of $V \in \mathbb{R}^{m \times d}$ be the $m$ vertices of $P$. Denote by $V^{\text {hog }}=(V, 1) \in \mathbb{R}^{m \times(d+1)}$ the homogenization of $V$. The column span of $V$ is a $(d+1)$-dimensional linear subspace. Choose $G \in \mathbb{R}^{m \times(m-d-1)}$ such that the columns form a basis for the orthogonal complement. Any such basis, regarded as an ordered collection of $m$ row vectors, is called a Gale transform of $P$. It is unique up to linear isomorphism and, by the reverse process, characterizes $V^{\text {hog }}$, again up to linear isomorphism. So it determines $P$ only up to a projective transformation. However, the striking feature of Gale transforms is that its combinatorial properties are, in a precise sense, dual to those of $P$; this correspondence goes by the name of Gale duality.

In order to state and work with Gale duality, we introduce some concepts and notation. As before, we write $V_{I}$ for the subset of the rows of $V$ indexed by $I \subseteq[\mathrm{m}]$. A subset $I \subset[m]$ names a coface of $P$ if the complement $V_{[m] \backslash I}$ is the vertex set of a face of $P$.

Definition 2.1 A collection of vectors $G=\left\{g_{1}, g_{2}, \ldots, g_{m}\right\} \subset \mathbb{R}^{k}$ is positively dependent if there are numbers $\lambda_{1}, \lambda_{2}, \ldots, \lambda_{m}>0$ such that $\lambda_{1} g_{1}+\cdots+\lambda_{m} g_{m}=0$. It is positively spanning if in addition $G$ is of full rank $k$.

"Being positively spanning" is, like "being spanning," an open condition, i.e., preserved under (sufficiently small) perturbations of the elements of $G$. This, however, 
is not true for "being positively dependent;" consider, e.g., $\{g,-g\}$ for $g \in \mathbb{R}^{k}, g \neq 0$, $k>1$.

Theorem 2.2 Gale duality Let $P=\operatorname{conv} V$ be a polytope and $G$ a Gale transform of $P$. Let $I \subset[m]$. Then $I$ names a coface of $P$ if and only if $G_{I}$ is positively dependent.

In light of Gale duality, the preceding theorem implies that, for a general polytope, not every subset of the vertex set of a face necessarily forms a face. This, however, is true for simplicial polytopes and, in fact, characterizes them. A still stronger condition is satisfied if no $d+1$ vertices of a $d$-polytope lie on a hyperplane, that is, if the vertices are in general position with respect to affine hyperplanes. This translates into Gale diagrams as follows.

Proposition 2.3 Let $P \subset \mathbb{R}^{d}$ be a polytope and $G \subset \mathbb{R}^{k}$ a Gale transform of $P$. Then $P$ is simplicial with vertices in general position if and only if the rows of $G$ are in general position with respect to linear hyperplanes, that is, if any $k$ vectors of $G$ are linearly independent.

\subsection{Faces Strictly Preserved by a Projection}

Projections are fundamental in polytope theory: Every polytope on $n$ vertices is the image of an $(n-1)$-simplex under an affine projection. This in particular says that the analysis of the images of polytopes under projection is as difficult as the general classification of all combinatorial types of polytopes. The problem is that a $k$-face $F \subset P$ can behave in various ways under projection: It can map to a $k$-face, or to part of a $k$-face, or to a lower-dimensional face of $\pi(P)$. Even if it maps to a $k$-face $\pi(F) \subset \pi(P)$, there may be other $k$-faces of $P$ that map to the same face $\tilde{F}=\pi(F)$. In that case, the face $\pi^{-1}(\tilde{F})$ has higher dimension than $F$. Thus, as a serious simplifying measure, we restrict our attention in the following to the most convenient situation of faces that are "strictly preserved" by a projection.

Definition 2.4 (Strictly preserved faces [18]) Let $P$ be a polytope and $Q=\pi(P)$ the image of $P$ under an affine projection $\pi: P \rightarrow \mathbb{R}^{d}$. A nonempty face $F$ of $P$ is (strictly) preserved by $\pi$ if:

(i) $\pi(F)$ is a face of $Q$ combinatorially equivalent to $F$, and (preserved face)

(ii) the preimage $\pi^{-1}(\pi(F))$ is $F$ (strictly preserved).

Since in the following we will be concerned exclusively with the analysis of strictly preserved faces, we will generally drop the modifier "strictly" starting now.

The following lemma gives an algebraic way to read off the preserved faces from a polytope in exterior presentation. Every affine projection $\pi: \mathbb{R}^{n} \rightarrow \mathbb{R}^{d}$ factors as an affine transformation followed a projection $\pi_{d}: \mathbb{R}^{n-d} \times \mathbb{R}^{d} \rightarrow \mathbb{R}^{d}$ that deletes the first $n-d$ coordinates, that is, $\pi_{d}(\bar{x}, \overline{\bar{x}})=\overline{\bar{x}}$ for all $(\bar{x}, \overline{\bar{x}}) \in \mathbb{R}^{n-d} \times \mathbb{R}^{d}$. Therefore, we will focus on the projections $\pi_{d}$ "to the last $d$ coordinates." For a polytope $P=$ $P(A, b) \subset \mathbb{R}^{n}$ in exterior presentation, the projection map $\pi_{d}$ naturally partitions the columns of $A$ as $A=(\bar{A} \mid \overline{\bar{A}})$. 
Lemma 2.5 (Projection Lemma: matrix version) Let $P=P(A, b) \subset \mathbb{R}^{n}$ be a polytope, $F$ a nonempty face of $P$, and $I=\mathrm{eq} F$ the index set of the inequalities that are tight at $F$. Then $F$ is preserved by the projection $\pi_{d}: P \rightarrow \mathbb{R}^{d}$ to the last $d$ coordinates if and only if the rows of $\bar{A}_{I}$ are positively spanning.

The proof makes use of the following geometric version of the Farkas Lemma.

Lemma 2.6 [16, Sect. 1.4] Let $P=P(A, b)$ be a polytope and $F \subseteq P$ a nonempty face. For a linear functional $\ell(x)=c x$, we denote by $P^{\ell}$ the nonempty face of $P$ on which $\ell$ attains its maximum. The linear function $\ell$ singles out $F$, that is, $P^{\ell}=F$, if and only if $c$ is a strictly positive linear combination of the rows of $A_{\mathrm{eq} F}$.

Proof of Lemma 2.5 We split the proof into two parts.

Claim $1 \tilde{F}=\pi_{d}(F)$ is a face of $\tilde{P}=\pi_{d}(P)$ with $\pi_{d}^{-1}(\tilde{F}) \cap P=F$ iff $\bar{A}_{I}$ is positively dependent.

By Lemma 2.6 the rows of $\bar{A}_{I}$ are positively dependent if and only if there is some $c \in \mathbb{R}^{d}$ such that the linear function $\ell(x):=(0, c) x=c \overline{\bar{x}}$ satisfies $P^{\ell}=F$. Rewriting $\ell=h \circ \pi_{d}$ with $h(\overline{\bar{x}})=c \overline{\bar{x}}$, we see that such $c$ exists if and only if there is a linear function $h$ on $\tilde{P}$ such that $\tilde{P}^{h}=\tilde{F}$.

Claim 2 Considering $F$ as a (sub-)polytope in its own right, then $\tilde{F}=\pi_{d}(F)$ is combinatorially equivalent to $F$ if and only if $A_{I}$ has full row rank.

The polytopes $F$ and $\tilde{F}$ are combinatorially equivalent iff they are affinely isomorphic. This happens if and only if the linear map $\pi_{d}$ is injective restricted the linear space $L=\left\{x: A_{I} x=0\right\}$, which is parallel to aff $F=\left\{x: A_{I} x=b_{I}\right\}$, the affine hull of $F$. Now, $\left.\pi_{d}\right|_{L}$ is injective iff ker $\pi_{d} \cap L \cong\left\{\bar{x}: \bar{A}_{I} \bar{x}=0\right\}$ is trivial.

See [14] for a proof in a different wording.

Lemma 2.5 allows us to guarantee that in certain situations every single $k$-face is preserved by a projection $\pi: P \rightarrow \pi(P)$. Then, however, we want to also see that $\pi(P)$ has no other $k$-face than those induced by the projection. This will be argued via the following lemma.

Lemma 2.7 Let $P=P(A, b) \subset \mathbb{R}^{n}$ be an $n$-polytope such that, for every vertex $v \in$ vert $P$, the rows of the matrix $\bar{A}_{\mathrm{eq}} v$ are in general position with respect to linear hyperplanes. Then every proper face of $P$ is either preserved under $\pi_{d}$, or its image under $\pi_{d}$ is not a face of $\pi_{d}(P)$.

Proof If $G \subset \mathbb{R}^{k}$ is a set of at least $k$ vectors in general position with respect to linear hyperplanes, then $\operatorname{dim} \operatorname{span} G^{\prime} \geq \min \left\{\left|G^{\prime}\right|, k\right\}$ for every subset $G^{\prime} \subseteq G$. In particular, every positively dependent subset is positively spanning.

Let $F \subset P$ be a proper face. From the proof of Lemma 2.5 it follows that $\pi_{d}(F)$ is a face iff $\bar{A}_{\text {eq } F}$ is positively dependent. Let $v \in$ vert $P$ be a vertex with $v \in F$. Then $\bar{A}_{\text {eq } F} \subseteq \bar{A}_{\text {eq } v}$, and $\bar{A}_{\text {eq } v} \subset \mathbb{R}^{n-d}$ is a set of at least $n$ vectors in general position with respect to linear hyperplanes. 
Corollary 2.8 If all $k$-faces of $P$ are preserved by the projection $\pi: P \rightarrow \pi(P)$, then all $k$-faces of $\pi(P)$ arise as images of $k$-faces of $P$.

Proof For any $k$-face $G \subseteq \pi(P)$, we know that $\widehat{G}=\pi_{d}{ }^{-1}(G)$ is a face of $P$ of dimension $\operatorname{dim} \widehat{G} \geq k$. Now if $F \subseteq \widehat{G}$ is any $k$-face of $\widehat{G}$, then by Lemma 2.7 either $F$ is preserved, and we get $\pi_{d}(F)=G$, or $F$ is not mapped to a face. The latter case cannot arise here.

\subsection{Generalized Deformed Products}

The orthogonal product $P \times Q \subset \mathbb{R}^{d+e}$ of a $d$-polytope $P=P(A, a) \subset \mathbb{R}^{d}$ and an $e$-polytope $Q=P(B, b) \subset \mathbb{R}^{e}$ is given in inequality description by the block diagonal system

$$
\begin{aligned}
A x \quad & \leq a, \\
B y & \leq b .
\end{aligned}
$$

We get a deformed product (with the combinatorial structure of the orthogonal product) if we generalize this into a block lower-triangular system, provided that $Q$ is simple and that we rescale the right-hand side of the system suitably.

Definition 2.9 (Rank $r$ deformed product) Let $P=P(A, a) \subset \mathbb{R}^{d}$ be a $d$-polytope and $Q=P(B, b) \subset \mathbb{R}^{e}$ a simple $e$-polytope with $A \in \mathbb{R}^{k \times d}$ and $B \in \mathbb{R}^{n \times e}$. Let $C \in \mathbb{R}^{n \times d}$ be an arbitrary matrix of rank $r$, and let $M \gg 0$ be large. The rank $r$ deformed product $P \bowtie_{C} Q \subset \mathbb{R}^{d+e}$ of $P$ and $Q$ with respect to $C$ is given by

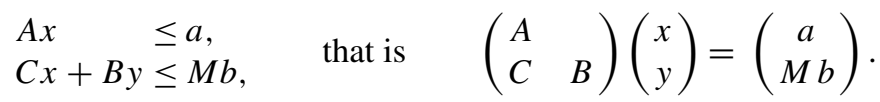

Proposition 2.10 Let $P=P(A, a) \subset \mathbb{R}^{d}$ be a d-polytope, $Q=P(B, b) \subset \mathbb{R}^{e}$ a simple e-polytope, $P \bowtie_{C} Q$ their deformed product, and $M>0$ the parameter involved. If $M$ is sufficiently large (depending on $B, b$, and $C$ ), then $P \bowtie_{C} Q$ and $P \times Q$ are combinatorially equivalent.

Our proposition may also be obtained from the Isomorphism Lemma [1, Lemma 2.4] that was applied by Amenta and Ziegler to prove the corresponding statement for (rank 1) deformed products. However, we use it in a dual form as given below. Again, for $I \subseteq[n]$, we write $P_{I}=P \cap\left\{x: A_{I} x=b_{I}\right\}$ for the smallest face $F \subseteq P$ that satisfies $I \subseteq$ eq $F$.

Lemma 2.11 (Isomorphism Lemma; dual formulation) Let $P=P(A, a)$ and $Q=P(B, b)$ be two polytopes with $n$ facets and $\operatorname{dim} P \geq \operatorname{dim} Q$. If

$$
P_{I} \text { is a vertex } \quad \Longrightarrow \quad Q_{I} \text { is nonempty }
$$

for every set $I \subset[n]$, then $P$ and $Q$ are of the same combinatorial type. 
Proof of Proposition 2.10 Since $Q$ is a simple polytope, we can find $M \gg 0$ such that $Q \cong P(B, M b-C v)$ for every $v \in$ vert $P$. In particular, if $u \in$ vert $Q$ is a vertex with $I=$ eq $u$, then $P(B, M b-C v)_{I}$ is a vertex. Thus, by the dual Isomorphism Lemma, the result follows.

Proposition 2.10 frees us from a discussion of right-hand sides. Therefore all deformed products hereafter are understood with a suitable right-hand side.

To see that the above definition of rank $r$ deformed products generalizes the (rank 1) deformed products of Amenta and Ziegler [1], we recall their $\mathcal{H}$-description of a deformed product. Let $P=P(A, a) \subset \mathbb{R}^{d}$ be a polytope and $\varphi: P \rightarrow \mathbb{R}$ an affine functional with $\varphi(P) \subseteq[0,1]$. Let $Q_{1}, Q_{2} \subset \mathbb{R}^{e}$ be "normally equivalent" $e$ polytopes, that is, combinatorially equivalent polytopes with the same left-hand side matrix, $Q_{i}=P\left(B, b_{i}\right)$ for $i=1,2$. Then, according to [1, Theorem 3.4(iii)], the exterior representation of $(P, \varphi) \bowtie\left(Q_{1}, Q_{2}\right)$ of the AZ-deformed product is given by

$$
(P, \varphi) \bowtie\left(Q_{1}, Q_{2}\right)=\left\{(x, y) \in \mathbb{R}^{d+e}: A x \leq a, B y \leq b_{1}-\left(b_{1}-b_{2}\right) \varphi(x)\right\} .
$$

Proposition 2.12 The AZ-deformed product is a rank 1 deformed product.

Proof Let $\varphi(x)=c^{\top} x+\delta$ be the affine functional. Let $C=\left(b_{1}-b_{2}\right) c^{\top}$ be the matrix of rank at most 1 with entries $C_{i j}:=\left(b_{1}-b_{2}\right)_{i} \cdot c_{j}$. Further, let $b=b_{1}-$ $\delta\left(b_{1}-b_{2}\right)$ and $Q=P(B, b)$. Now, rewriting the inequality system for $(P, \varphi) \bowtie$ $\left(Q_{1}, Q_{2}\right)$ proves the claim.

\section{Neighborly Cubical Polytopes}

For $\varepsilon>0$, the interval $I_{\varepsilon}=\{x \in \mathbb{R}: \pm \varepsilon x \leq 1\}$ is a one-dimensional, simple polytope. Its poset of nonempty faces is the poset on $\{+,-, 0\}$ with order relations $+\prec 0$ and $-\prec 0$. The signs \pm represent the vertices of the interval with the suggestive notation that \pm names the vertices given by $\pm \varepsilon x=1$, while 0 stands for the unique (improper) one-dimensional face. An $n$-fold product of intervals gives a combinatorial $n$-dimensional cube $C_{n}$ with inequality system

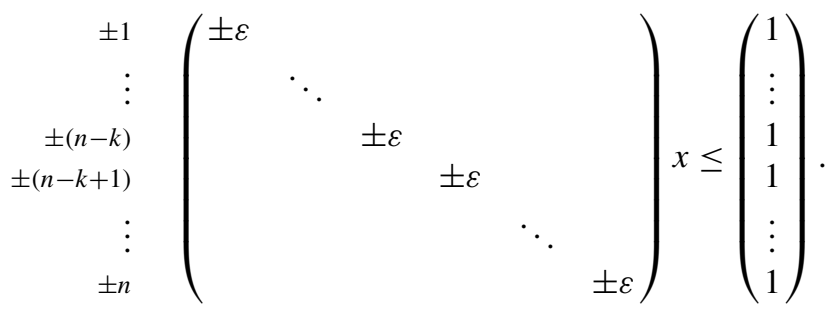

Every row in the above system represents two inequalities: The $i$ th row prescribes an upper and a lower bound for the variable $x_{i}$. Left to the system are the labels of the rows to which we will refer in the following.

On the level of posets the facial structure is captured by an $n$-fold direct product of the poset above. The nonempty faces of $C_{n}$ correspond to the elements of $\{+,-, 0\}^{n}$ 
with the (component-wise) induced order relation. An element $\gamma \in\{+,-, 0\}^{n}$ represents the unique face $F_{\gamma}$ with equality set eq $F_{\gamma}=\left\{\gamma_{i} i: i \in[n]\right\}$ of dimension $\operatorname{dim} F_{\gamma}=\#\left\{i \in[n]: \gamma_{i}=0\right\}$. This, in particular, gives the $f$-vector as $f_{i}\left(C_{n}\right)=$ $\left(\begin{array}{c}n \\ i\end{array}\right) 2^{n-i}$.

The cube, as an iterated product of simple 1-polytopes, lends itself to deformation beneath the "diagonal" that yields, figuratively, a deformed product of intervals. In the following we construct deformed cubes that all subscribe to the same deformation scheme. To avoid cumbersome descriptions, we fix a template for a deformed cube.

Definition 3.1 (Deformed Cube Template) For $n \geq d \geq 2$, let $G=\left\{g_{1}, \ldots, g_{d-1}\right\} \subset$ $\mathbb{R}^{n-d}$ be an ordered collection of row vectors, and let $\varepsilon>0$. We denote by $C_{n}(G)$ the deformed cube with lhs matrix

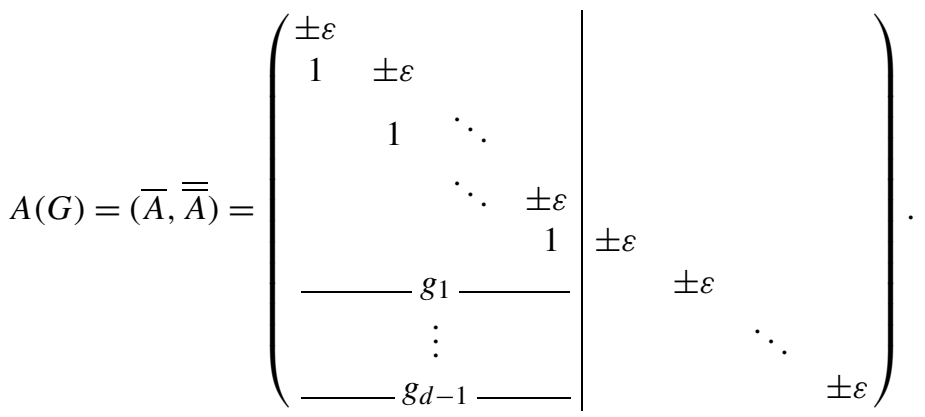

Proposition 2.10 assures of a suitable right-hand side such that $C_{n}(G)$ is a combinatorial $n$-cube. Up to this point, we required $\varepsilon$ to be nothing but positive; this will be subject to change, soon.

The polytope we are striving for is the image of $C_{n}(G)$ under projection. Recall that our projections will be onto the last $d$ coordinates for which the vertical bar in (1) is a reminder.

We now come to the first main result of this section.

Theorem 3.2 (Joswig and Ziegler [7, Theorem 17]) For every $2 \leq d \leq n$, there is a cubical d-polytope whose $\left(\left\lfloor\frac{d}{2}\right\rfloor-1\right)$-skeleton is isomorphic to that of an $n$-cube.

Proof Let $Q$ be a neighborly simplicial $(d-2)$-polytope with $n-1$ vertices in general position. In particular, $Q$ has the property that every subset of at most $\left\lfloor\frac{d-2}{2}\right\rfloor=\left\lfloor\frac{d}{2}\right\rfloor-1$ vertices forms a face of $Q$. For an arbitrary but fixed ordering of the vertices, let $G \in \mathbb{R}^{(n-1) \times(n-d)}$ be a Gale transform of $Q$. As the vertices of $Q$ are in general position, we can choose a Gale transform of the form $G=\left(\begin{array}{c}I_{n-d} \\ \bar{G}\end{array}\right)$, where $\bar{G}=\left\{g_{1}, \ldots, g_{d-1}\right\} \subset \mathbb{R}^{n-d}$ is an ordered collection of row vectors. Let $C=C_{n}(\bar{G})$ be the deformed cube given by the template (1) with respect to $\bar{G}$.

We claim that the projection of $C$ to the last $d$ coordinates yields the result. For this, we prove that all faces of dimension up to $k=\left\lfloor\frac{d}{2}\right\rfloor-1$ survive the projection. In order to do so, we propose the following strategy: We will show that, for an arbitrary vertex $v$ of $C$, the incident faces of dimension $\leq k$ are retained. 
Consider $\bar{A}_{\text {eq } v}$, the first $n-d$ columns of the inequalities of (1) which are tight at $v$. The matrix is of the form

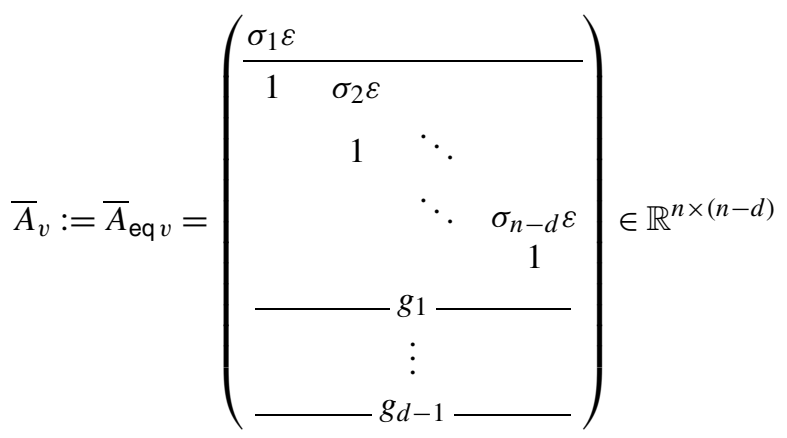

with $\sigma_{1}, \ldots, \sigma_{n-d} \in\{+,-\}$.

Since the vertices of $Q$ are in general position, by Proposition 2.3, $G$ is a configuration of vectors in general position with respect to linear hyperplanes. Thus, for $\varepsilon>0$ sufficiently small, $\bar{A}_{v}$ take away the first row is still the Gale transform of a polytope combinatorially equivalent to $Q$. By Gale duality, this in particular means that discarding up to $\left\lfloor\frac{d-2}{2}\right\rfloor=k$ rows from $\bar{A}_{v}$ leaves the remaining ones positively spanning.

Now, let $F \subset C$ be a face of dimension $\ell \leq k$ with $v \in F$. By the Projection Lemma $2.5, F$ is strictly preserved by the projection iff the rows of $\bar{A}_{I}$ for $I=$ eq $F$ are positively spanning. Since $C$ is simple, $\bar{A}_{I}$ is an $(n-\ell)$-rowed submatrix of $\bar{A}_{\text {eq } v}$, that is, at most $k$ rows have been discarded from $\bar{A}_{v}$.

Choosing $\varepsilon$ sufficiently small also has the effect that the rows of $\bar{A}_{v}$ are in general position with respect to linear hyperplanes. Thus, Corollary 2.8 vouches for the fact that all faces of $\pi_{d}\left(C_{n}(\bar{G})\right)$ arise from the projection of $C_{n}(\bar{G})$.

The polytope $\pi_{d}\left(C_{n}(\bar{G})\right)$ constructed in the course of the proof depends on the choice of a neighborly simplicial $(d-2)$-polytope $Q$ with $n-1$ vertices in general position, equipped with an ordering of its vertices. In particular, the order of the vertices is needed to determine $\bar{G}$ and thus $C_{n}(\bar{G})$. Nevertheless, by abuse of notation we will write $C_{n}(Q)$ for the deformed cube $C_{n}(\bar{G})$. We will see in the next section that, in fact, the combinatorics of $\pi_{d}\left(C_{n}(Q)\right)$ is determined by the choice of $Q$ and the vertex order. In Sect. 3.2, we show that the polytopes constructed in [7] correspond to the case where $Q$ is a cyclic polytope with the standard vertex ordering. According to Joswig and Rörig [6], they have the same combinatorial types as the spheres originally constructed by Babson, Billera, and Chan [2]. For now, we baptize the polytope that we have constructed.

Definition 3.3 For $n \geq d \geq 2$ and a neighborly simplicial $(d-2)$-polytope $Q$ on $n-1$ ordered vertices in general position, we denote the neighborly cubical polytope $\pi_{d}\left(C_{n}(Q)\right)$ by $\mathrm{NCP}_{n, d}(Q)$.

Let us briefly comment on the extremal choices of $d$. For $d=n$, the polytope $\mathrm{NCP}_{n, n}(Q)$ is combinatorially isomorphic to an $n$-cube. The neighborly simplicial polytope $Q$ is then an $(n-2)$-polytope with $n-1$ vertices, a simplex. For 
$d=2$, the polytope $\mathrm{NCP}_{n, 2}(Q)$ is a $2^{n}$-gon, and $C_{n}(Q)$ is, in fact, a realization of a Goldfarb cube [4]. Note that for this the "neighborly simplicial polytope" in question is a 0 -dimensional polytope consisting of $n-1$ points. The Gale transform of such a polytope/configuration is given by the vertices of an $(n-2)$-simplex with vertices $\left\{e_{1}, e_{2}, \ldots, e_{n-2},-\mathbb{1}\right\}$.

The proof can be adapted to yield a $k$-neighborly cubical polytope, that is, a polytope having its $k$-skeleton isomorphic to that of an $n$-cube. By [7, Corollary 5], the neighborliness is bounded by $k \leq\left\lfloor\frac{d}{2}\right\rfloor-1$. In our construction this fact is reflected as follows. The polytope $\mathrm{NCP}_{n, d}(Q)$ is $k$-neighborly cubical iff $Q$ is $k$-neighborly simplicial. By [16, Exercise 0.10], neighborliness for $(d-2)$-polytopes is bounded by $\left\lfloor\frac{d-2}{2}\right\rfloor$.

\subsection{Combinatorial Description of the Neighborly Cubical Polytopes}

We describe the face lattice of $\mathrm{NCP}_{n, d}(Q)$ in terms of lexicographic triangulations of $Q$. We start by giving the necessary background on regular subdivisions with an emphasis on lexicographic triangulations in terms of Gale transforms. Our main sources are the paper by Lee [9] and the (upcoming) book by De Loera et al. [3].

Let $Q$ be a simplicial $D$-dimensional simplicial polytope for $D=d-2$ on $N=n-1$ ordered vertices. We further assume that the vertices of $Q$ are in general position, i.e., all vertex-induced subpolytopes are simplicial as well. Let the rows of $V \in \mathbb{R}^{N \times D}$ be the vertices of $Q$ in some ordering, and let $\omega=\left(\omega_{1}, \ldots, \omega_{N}\right)^{\top} \in \mathbb{R}^{N}$ be a set of heights. Denote by $V^{\omega}=(\omega, V) \in \mathbb{R}^{N \times(D+1)}$ the ordered set of lifted vertices $\left(\omega_{i}, v_{i}\right)$ for $i=1, \ldots, N$. Let $a=\left(\omega_{0}, v_{0}\right) \in \mathbb{R}^{D+1}$ be arbitrary with $\omega_{0} \gg$ $\max _{i}\left|\omega_{i}\right|$ and consider the polytope $Q^{\omega}=\operatorname{conv}\left(V^{\omega} \cup a\right)$. If $\omega_{0}$ is sufficiently large, then the vertex figure of $a$ in $Q^{\omega}$ is isomorphic to $Q$, and the closed star of $a$ in $\partial Q^{\omega}$ is isomorphic to that of the apex of a pyramid over $Q$. The anti-star (or deletion) of $a$ in the boundary of $Q^{\omega}$, i.e., the faces of $Q^{\omega}$ not containing $a$, constitute a pure $D$-dimensional polytopal complex $\Gamma_{\omega}$, the $\omega$-induced (or $\omega$-coherent) subdivision. The name "subdivision" stems from the fact that the underlying set $\left\|\Gamma_{\omega}\right\|$ is piecewise-linear homeomorphic to $Q$ via the projection onto the last $D$ coordinates. The inclusion maximal polytopes in $\Gamma_{\omega}$ are called cells. $\Gamma_{\omega}$ is called a triangulation if every cell is a $D$-simplex. Altering the heights $\omega_{i}^{\prime}=\omega_{i}+\ell\left(v_{i}\right)$ along an affine functional $\ell: Q \rightarrow \mathbb{R}$ leaves the induced subdivision unchanged. We call a set of heights normalized if its support is minimal in the corresponding equivalence class.

Proposition 3.4 Let $\omega^{\top}=\left(\omega_{1}, \ldots, \omega_{N-D-1}, 0, \ldots, 0\right) \in \mathbb{R}^{N}$ be a normalized set of heights, and let $G=\left(\begin{array}{c}\operatorname{ld}_{N-D-1} \\ \bar{G}\end{array}\right) \in \mathbb{R}^{N \times(N-D-1)}$. For $\varepsilon>0$ sufficiently small, the matrix

$$
G_{\omega}=\left(\begin{array}{c}
-\varepsilon \bar{\omega} \\
G
\end{array}\right) \in \mathbb{R}^{(N+1) \times(N-D-1)}
$$

with $\bar{\omega}=\left(\omega_{1}, \ldots, \omega_{n-d-1}\right)$ is a Gale transform of a polytope combinatorially equivalent to $Q^{\omega}$. 
Proof It is easily verified that the columns of

$$
\left(\begin{array}{ccc}
1 & 1 & \mathbb{O} \\
\mathbb{1}+\varepsilon \omega & \varepsilon \omega & V
\end{array}\right) \in \mathbb{R}^{(N+1) \times(D+2)}
$$

form a basis for the orthogonal complement of the column span of $G_{\omega}$. For $\varepsilon$ sufficiently small, the first column is strictly positive, and dehomogenizing with respect to this column yields the desired polytope.

In particular, $G_{\omega}$ encodes the combinatorial structure of $Q$ and that of the $\omega$-induced regular subdivision.

Consider the two induced regular subdivisions of $Q$ obtained by lifting the vertex $v_{1}$ to height $\omega_{1}= \pm h$ with $h>0$ and fixing all the remaining heights to 0 . In both cases the lifted polytope is a pyramid over the polytope $Q^{\prime}=\operatorname{conv}\left(V \backslash v_{1}\right)$. For $\omega_{1}=-h$, the subdivision is said to be obtained by pulling $v_{1}$, and its cells are pyramids over the remote facets of $Q^{\prime}$, that is, the facets common to both $Q$ and $Q^{\prime}$. This subdivision is, in fact, a triangulation since its cells are pyramids over $(D-1)$ simplices. The other subdivision $(\omega=+h)$ is said to be obtained by pushing $v_{1}$, and its cells are pyramids over the newly created facets of $Q^{\prime}$, which are again simplices, plus one (possibly nonsimplex) cell that is $Q^{\prime}$.

The ordering of the vertices of $Q$ gives rise to a chain of (sub-)polytopes $Q=Q_{0} \supset Q_{1} \supset \cdots \supset Q_{N-D-1}=\Delta_{D}$ with $Q_{i}=$ conv $\left\{v_{i+1}, \ldots, v_{N}\right\}$ simplicial $D$-polytopes. Let $1 \leq k \leq N-D-1$, then the kth lexicographic triangulation Lex $\operatorname{Le}_{k}$ of $Q$ in the given vertex order is the triangulation obtained by pushing the first $k-1$ vertices in the given order and then pulling the $k$ th vertex. That is to say, pushing $v_{1}$ creates a subdivision of $Q=Q_{0}$ that has $Q_{1}$ as its only nonsimplex cell. Subsequently, the cell $Q_{1}$ gets replaced by a pushing subdivision of $Q_{1}$ with respect to $v_{2}$, and so on. Finally, pulling $v_{k+1}$ in $Q_{k}$ completes the triangulation. The following lemma asserts that the above procedure yields a regular subdivision by giving a description in the spirit of Proposition 3.4.

Lemma 3.5 ([9, Example 2], [13]) Let $\varepsilon>0$ and $\omega=\left(\omega_{1}, \omega_{2}, \ldots, \omega_{N-D-1}, 0\right.$, $\ldots, 0) \in \mathbb{R}^{N}$ be a set of normalized heights satisfying $\left|\omega_{i+1}\right| \leq \varepsilon\left|\omega_{i}\right|$ for all $1 \leq$ $i \leq N-D-2$. If $\varepsilon>0$ is sufficiently small, then $G_{\omega}$ is a Gale transform encoding $\operatorname{Lex}_{k} Q$ for

$$
k=\min \left\{i: \omega_{i}<0\right\} \cup\{n-d-1\} .
$$

Definition 3.6 We call the polytope $\mathcal{L}_{k}(Q)=\tilde{Q}^{\omega}$ corresponding to $G_{\omega}$ the $k$ th lexicographic pyramid of $Q$.

According to the remarks following Proposition 3.4, $\mathcal{L}_{k}(Q)$ carries the combinatorics of $Q$ as well as that of $\operatorname{Lex}_{k} Q$. So every facet of $\mathcal{L}_{k}(Q)$ is either a pyramid over a facet of $Q$ or a cell of $\operatorname{Lex}_{k} Q$.

We are now in a position to determine the combinatorics of $\mathrm{NCP}_{n, d}(Q)$. To be more precise, we determine the local combinatorial structure, i.e., for any given vertex, we describe the set of facets that contain it. The construction of a neighborly 
cubical polytope depended on an ordering of the vertices of $Q$, which we fix for the following theorem.

Theorem 3.7 Let $C=C_{n}(Q)$ be the deformed cube with respect to $Q$. Further, let $v \in C$ be an arbitrary vertex with eq $v$ given by $\sigma \in\{+,-\}^{n}$. Then the vertex figure of $\pi_{d}(v)$ in $\mathrm{NCP}_{n, d}(Q)$ is isomorphic to $\mathcal{L}_{p}(Q)$ for

$$
p=\min \left\{i \in[n]: \sigma_{i}=+\right\} \cup\{n-d-1\} .
$$

In particular, the $(d-1)$-faces of $C$ containing $v$ that are preserved by projection are in one-to-one correspondence to the facets of $\mathcal{L}_{p}(Q)$.

Proof After a suitable base transformation of (2) by means of column operations, the first $n-d$ columns of $A_{\text {eq } v}$ can be assumed to be of the form

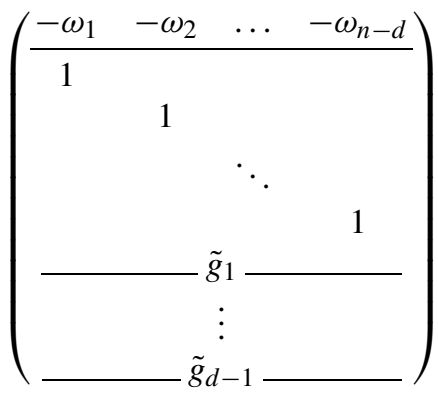

with

$$
\omega_{i}=(-1)^{i} \varepsilon^{i} \prod_{j=1}^{i} \sigma_{j} .
$$

By Lemma 3.5, this is a Gale transform of $\mathcal{L}_{k}(Q)$ with $k=p$.

Any generic projection of polytopes $\pi: P \rightarrow P^{\prime}=\pi(P)$ induces a (contravariant) order and rank preserving map $\pi^{\#}: \mathcal{F} \partial P^{\prime} \hookrightarrow \mathcal{F} \partial P$.

The face poset of $\partial \mathrm{NCP}_{n, d}(Q) / u$, the boundary complex of the vertex figure of $u=\pi_{d}(v)$ in $\operatorname{NCP}_{n, d}(Q)$, is isomorphic to $\pi^{\#}\left(\mathcal{F} \partial \mathrm{NCP}_{n, d}(Q)_{\geq u}\right)$, the image of the principal filter of $u$. By the Projection Lemma, the image coincides with the embedding of $\mathcal{L}_{p}(Q)$ into the vertex figure $\mathcal{F} \partial C_{n}(Q)_{\geq v}$.

Theorem 3.7 implies that the quotient $\mathrm{NCP}_{n, d}(Q) / e$ with respect to certain edges is isomorphic to $Q$. This implies the following result, which is already implicit in [2]; see also [6, Theorem 3.9].

Corollary 3.8 Nonisomorphic neighborly simplicial $(d-2)$-polytopes $Q$ and $Q^{\prime}$ yield nonisomorphic neighborly cubical polytopes $\mathrm{NCP}_{n, d}(Q)$ and $\mathrm{NCP}_{n, d}\left(Q^{\prime}\right)$. Moreover, there are at least as many different combinatorial types of $d$-dimensional neighborly cubical polytopes as there are neighborly simplicial $(d-2)$-polytopes on $n-1$ vertices. 
The number of combinatorial types of neighborly simplicial polytopes is huge: see [15].

\subsection{Neighborly Cubical Polytopes from Cyclic Polytopes}

In this section we (re)construct the neighborly cubical polytopes of Joswig and Ziegler [7]. This specializes the discussion in the previous section to the case of $Q$ a cyclic polytope in the standard vertex ordering. By a thorough analysis of the lexicographic triangulations of cyclic polytopes, we recover the "cubical Gale's evenness criterion" of [7]. For a treatment of cyclic polytopes and their triangulations beyond our needs, we refer the reader to $[3,16]$.

The degree $D$ moment curve is given by $t \mapsto \gamma(t)=\left(t, t^{2}, \ldots, t^{D}\right) \in \mathbb{R}^{D}$. For given pairwise distinct values $t_{1}, t_{2}, \ldots, t_{N} \in \mathbb{R}$ with $N \geq D+1$, the convex hull of the corresponding points on the moment curve $\operatorname{Cyc}_{D}\left(t_{1}, \ldots, t_{N}\right)=\operatorname{conv}\left\{\gamma\left(t_{i}\right)\right.$ : $i \in[N]\}$ is a convex $D$-dimensional polytope. A fundamental consequence of the theorem below is that the combinatorial type of $\operatorname{Cyc}_{D}\left(t_{1}, \ldots, t_{N}\right)$ is independent of the actual values $t_{i}$. Therefore, we work with $\operatorname{Cyc}_{D}(N):=\operatorname{Cyc}_{d}(1,2, \ldots, N)$, the $D$-dimensional cyclic polytope on $N$ vertices in standard order. For the sake of notational convenience later on, we describe its faces in terms of characteristic vectors of cofaces: A vector $\alpha \in\{0,1\}^{N}$ names a coface of $\mathrm{Cyc}_{D}(N)$ iff $\operatorname{conv}\left\{\gamma(i): \alpha_{i}=0\right\}$ is a face of $\operatorname{Cyc}_{D}(N)$. We also extend the notion of "co-" to subdivisions and, therefore, speak freely about cocells.

Let $\alpha \in\{0,1\}^{N}$ be such that $\#\left\{j<i: \alpha_{j}=0\right\}$ has the same parity for every $i \in[N]$ with $\alpha_{i}=1$. Then $\alpha$ is called even or odd according to this parity.

Theorem 3.9 (Gale's Evenness Criterion [5, Sect. 4.7], [16, Theorem 0.7], [3, Theorem 6.2.6]) A vector $\alpha \in\{0,1\}^{N}$ names a cofacet of $\mathrm{Cyc}_{D}(N)$ if and only if $\alpha$ has exactly $D$ zero entries and is either even or odd.

As a byproduct, we get that cyclic polytopes are:

- simplicial, since all facets have exactly $D$ vertices;

- in general position, since every subpolytope is again cyclic;

- neighborly, since every $\alpha \in\{0,1\}^{N}$ with $\leq\left\lfloor\frac{D}{2}\right\rfloor$ zeros can be made to meet the above conditions by changing entries $1 \rightarrow 0$.

From a geometric point of view, the odd and even (co)facets correspond to the upper and lower facets of $\mathrm{Cyc}_{D}(N)$ with respect to the last coordinate. This dichotomy among the facets allows for an explicit characterization of the (simplicial) cells of a pushing/pulling subdivision of $\operatorname{Cyc}_{D}(N)$ with respect to the first vertex. Moreover, since every vertex-induced subpolytope of $\mathrm{Cyc}_{D}(N)$ is again cyclic, from this we will derive a complete description of the lexicographic triangulations of cyclic polytopes with vertices in standard order.

To prepare for the precise statement, let $Q=\operatorname{Cyc}_{D}(N)=\operatorname{conv}\left\{v_{i}=\gamma_{d}(i): i \in\right.$ $[N]\}$ and $Q^{\prime}=\operatorname{conv}\left\{v_{2}, \ldots, v_{N}\right\} \cong \operatorname{Cyc}_{D}(N-1)$ the subpolytope on all vertices except the first. Let $\Gamma$ be the subdivision of $Q$ obtained by pulling or pushing $v_{1}$. Any cell in $\Gamma$ that contains $v_{1}$ is a $D$-simplex and, therefore, let $\alpha \in\{0,1\}^{N}$ be a 
cocell with $D+1$ zero entries and $\alpha_{1}=0$. Indeed, any such cell is a pyramid over a facet of $Q^{\prime}$, and thus $\alpha$ is of the form $\alpha=\left(0, \alpha^{\prime}\right)$, and $\alpha^{\prime}$ adheres to the Gale's evenness criterion. The cocell $\alpha$ is part of a pushing or a pulling subdivision of $Q$ if and only if $\alpha$ is or is not a cofacet of $Q$. Clearly, the first gap in $\alpha$ is even, and, hence, the parity of the gaps of $\alpha^{\prime}$ concludes the characterization.

Lemma 3.10 Let $Q=\operatorname{Cyc}_{D}(N)$ be a cyclic polytope, and let $\mathcal{L}_{k}(Q)$ be a lexicographic pyramid of $Q$. Let $\alpha \in\{0,1\}^{N+1}$ with $D+1$ zero entries, and let $p=\min \{i$ : $\left.\alpha_{i}=0\right\}$. Thus $\alpha$ is of the form

$$
\alpha=(\underbrace{1,1, \ldots, 1}_{p-1}, 0, \alpha^{\prime}) .
$$

Then $\alpha$ is a cofacet of $\mathcal{L}_{k}(Q)$ if and only if one of the following conditions is satisfied:

(i) $1=p$, and $\alpha^{\prime}$ is a cofacet of $\mathrm{Cyc}_{d}(n)$;

(ii) $1<p<k$, and $\alpha^{\prime}$ is even;

(iii) $p=k$, and $\alpha^{\prime}$ is odd.

Proof Every facet containing the 0 th vertex is a pyramid over a facet of $Q$, and every incident facet is of the form $\alpha=\left(0, \alpha^{\prime}\right)$ with $\alpha^{\prime}$ a cofacet of $Q$.

If $2 \leq p<k$, then $\alpha$ names a cocell of the pushing subdivision of $Q_{p-1}=$ conv $\left\{v_{p}, \ldots, v_{N}\right\}$ with respect to $v_{p}$ and containing $v_{p}$. This, however, is the case if and only if $\alpha^{\prime}$ is an even cofacet of $Q_{p}$. The case $p=k$ follows from similar considerations.

Setting $N=n-1$ and $D=d-2$ and combining the above description with Theorem 3.7, we obtain the following result of Joswig and Ziegler.

Theorem 3.11 (Cubical Gale's Evenness Condition [7]) Let $F$ be a $(d-1)$-face of the deformed cube $C=C_{n}(Q)$ with $Q:=\mathrm{Cyc}_{d-2}(n-1)$. Let eq $F$ be given by $\alpha \in\{+,-, 0\}^{n}$, and let $p \geq 1$ be the smallest index such that $\alpha_{p}=0$. The face $F$ projects to a facet of $\mathrm{NCP}_{n, d}(Q)$ if and only if $\alpha$ is of the form

$$
\alpha=(\underbrace{-,-, \cdots,-}_{p-2}, \sigma, 0, \alpha^{\prime})
$$

with $\left|\alpha^{\prime}\right|=\left(\left|\alpha_{p+1}^{\prime}\right|, \ldots,\left|\alpha_{n}^{\prime}\right|\right) \in\{0,1\}^{n-p}$ satisfying the ordinary Gale's evenness condition and if, for $p>1$, one of the following conditions holds:

(i) $\sigma=-$, and $\left|\alpha^{\prime}\right|$ is even, or

(ii) $\sigma=+$, and $\left|\alpha^{\prime}\right|$ is odd.

Proof Let $v \in F \subset C$ be a vertex with equality set $\beta=$ eq $v$ and such that $\beta_{p}=+$. By Theorem 3.7, the vertex figure of $\pi_{d}(v)$ in $\operatorname{NCP}_{n, d}(Q)$ is isomorphic to $\mathcal{L}_{k}(Q)$, with $k \in\{p-1, p\}$.

Thus $F$ projects to a facet of $\operatorname{NCP}_{n, d}(Q)$ if and only if $|\alpha|$ is a cofacet of $\mathcal{L}_{k}(Q)$. The result now follows from Lemma 3.10 by noting that $k=p-1$ iff $\sigma=-$. 


\section{Deformed Products of Polygons}

The projected deformed products of polygons (PDPPs) are four-dimensional polytopes. They were constructed in [18] because of their extremal $f$-vectors: For these polytopes, the fatness parameter $\Phi(P):=\frac{f_{1}+f_{2}-20}{f_{0}+f_{3}-10}$ is large, getting arbitrarily close to 9 . This parameter, introduced in [17], is crucial for the $f$-vector theory of 4-polytopes. In [18] the $f$-vectors of the PDPPs were computed without having a combinatorial characterization of the polytopes in reach.

However, the PDPPs are yet another instance of projections of deformed products, so the theory developed here gives us a firm grip on their properties. In the following we generalize the construction to higher dimensions and analyze its combinatorial structure using the tools developed in this paper. In particular, a description of the facets of the PDPPs appears for the first time.

To begin with, the following is a generalization of Theorem 3.2.

Theorem 4.1 Let $m \geq 4$ be even. For every $2 \leq d \leq 2 r$, there is a $d$-polytope whose $\left(\left\lfloor\frac{d}{2}\right\rfloor-1\right)$-skeleton is combinatorially isomorphic to that of an $r$-fold product of m-gons.

Let us remark that the proofs of the results in this section can be adapted to yield the generalizations for products of even polygons with varying numbers of vertices in each factor. However, the generalized results require more technical and notational overhead. Therefore, we trade generality in for clarity and only give the uniform versions of the results.

For $m=4$, the $r$-fold product of quadrilaterals is actually a cube of dimension $n=2 r$, and thus $\mathrm{NCP}_{n, d}(Q)$ satisfies the claims made. In the inequality description the quadrilaterals can be seen by pairing up the intervals indicated by the framed submatrices below:

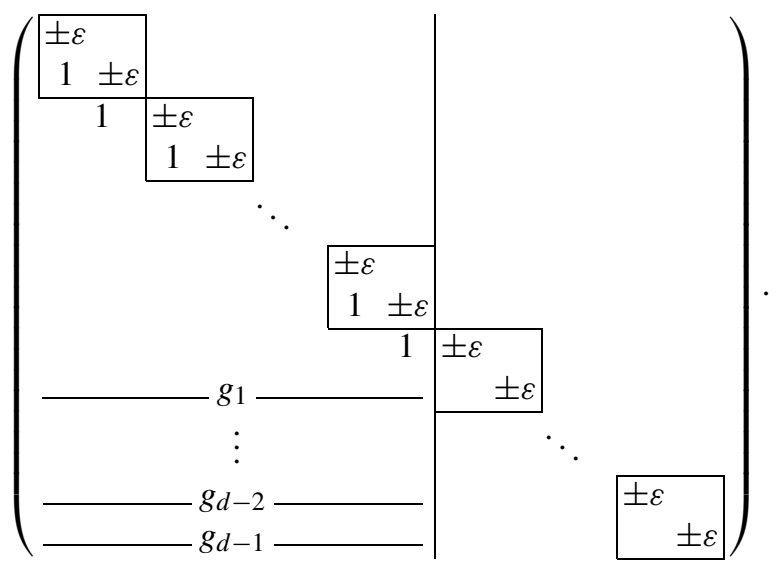


We wish to build on this special case and therefore consider the normals of such a quad:

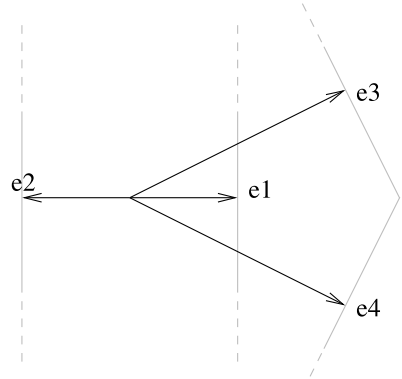

The polygons we are heading for arise as generalizations of the above quad. For $m \geq 4$ even, consider the vectors

$$
\begin{aligned}
& a_{0}=(-1,0), \\
& a_{i}=\left(1, \varepsilon \frac{m-2 i}{m-2}\right) \quad \text { for } i=1, \ldots, m-1,
\end{aligned}
$$

as shown below. For suitable $b_{0}, b_{1}, \ldots, b_{m-1}>0$,

$$
a_{i}^{\top} x \leq b_{i} \quad \text { for } i=0, \ldots, m-1
$$

describes a convex $m$-gon in the plane:

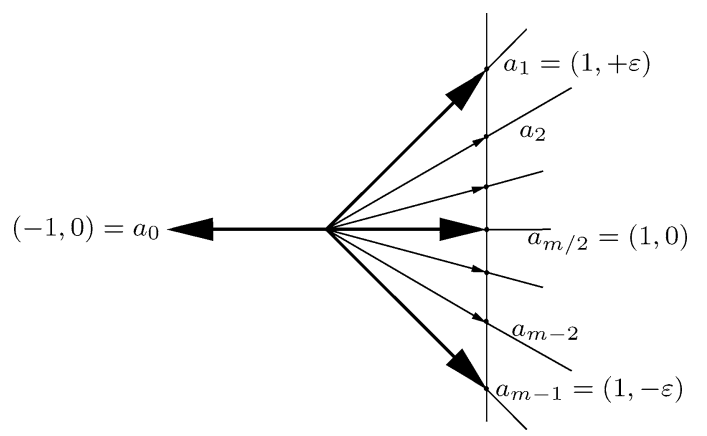

For the finishing touch, we scale every even-indexed inequality by $\varepsilon$,

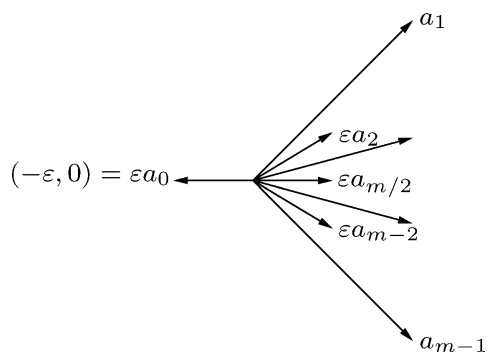


We arrange the scaled normals and right-hand sides into a matrix and vector, respectively,

$$
A=\left(\begin{array}{c}
\varepsilon a_{0} \\
a_{1} \\
\varepsilon a_{2} \\
\vdots \\
a_{m-1}
\end{array}\right) \quad \text { and } \quad b=\left(\begin{array}{c}
\varepsilon b_{0} \\
b_{1} \\
\varepsilon b_{2} \\
\vdots \\
b_{m-1}
\end{array}\right)
$$

Using these special polygons, we set up a template for a deformed product of polygons (DPP).

Definition 4.2 (DPP template) For $m \geq 4$ even and $2 r \geq d \geq 2$, let $G=\left\{g_{1}, \ldots\right.$, $\left.g_{d-1}\right\} \subset \mathbb{R}^{2 r-d}$ be an ordered collection of row vectors. We denote by $P_{2 r}(G ; m)$ the deformed product of polygons with lhs inequality system

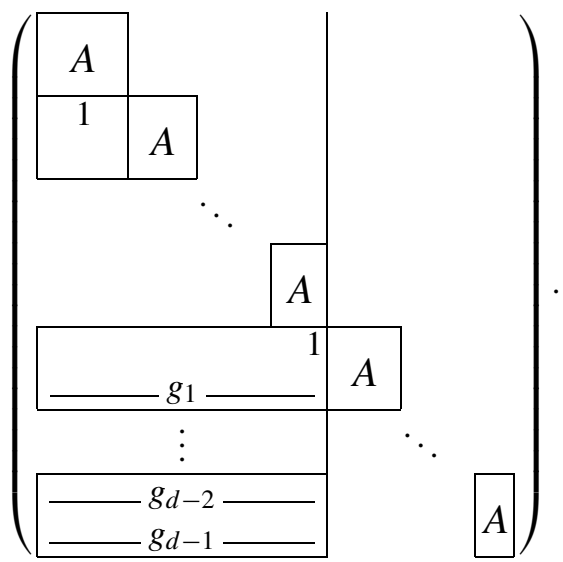

In the above inequality system, the framed blocks denote matrices of appropriate sizes that contain the depicted block repeated row-wise $\frac{m}{2}$ times. In particular,

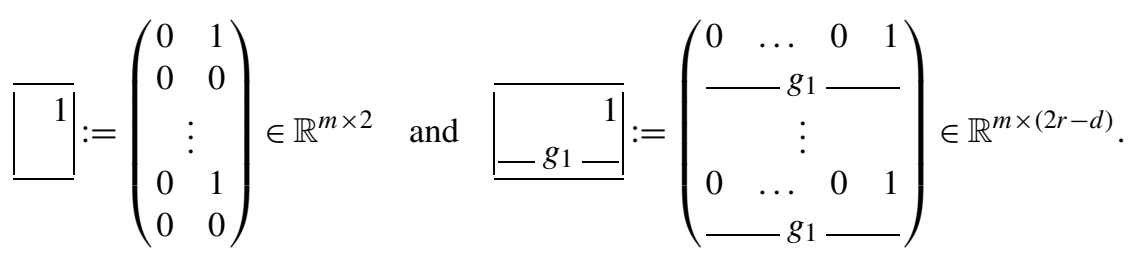

Proof of Theorem 4.1 Let $P=P_{2 r}(\bar{G} ; m)$ be the deformed product of $m$-gons according to the DPP template (3) which is determined by a Gale transform

$$
G=\left(\begin{array}{c}
I_{d-2} \\
\bar{G}
\end{array}\right)
$$

of a neighborly simplicial $(d-2)$-polytope $Q$ with $2 r-1$ ordered vertices in general position. Equipped with a suitable right-hand side, the polytope $P$ is an iter- 
ated rank 2 deformed product of polygons and thus combinatorially equivalent to the $r$-fold product of an $m$-gon.

Now for an arbitrary vertex $v$ of $P$, the matrix $\bar{A}_{\text {eq } v}$ is of the form

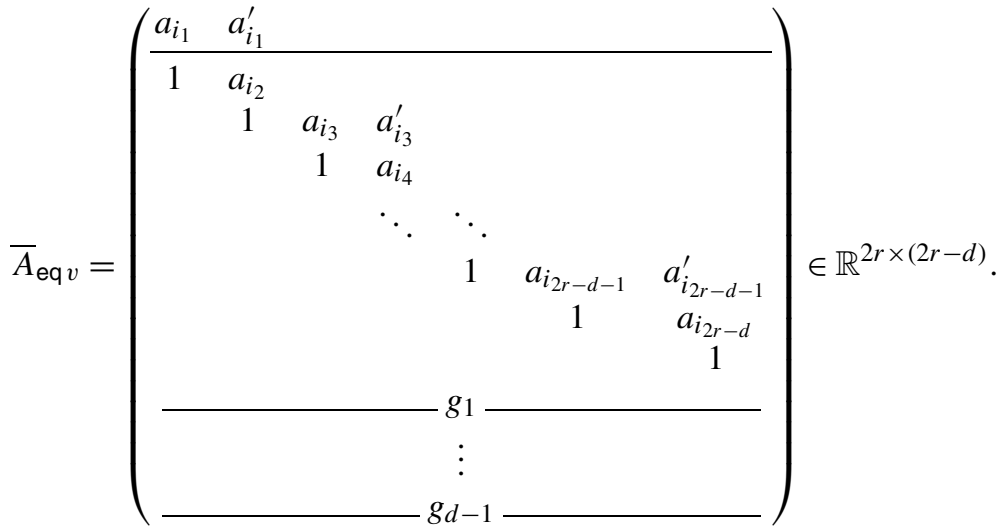

The equality set of a vertex $v$ is formed by two cyclically adjacent facets from each polygon in the product. This means, in particular, that from each polygon there is an even and an odd facet present in eq $v$. Every such pair is of the form

$$
\left(\begin{array}{cc}
a_{i_{\ell}} & a_{i_{\ell}}^{\prime} \\
1 & a_{i_{\ell+1}}
\end{array}\right) .
$$

The absolute values of the diagonal entries are bounded by $\varepsilon$, while $\left|a_{i_{\ell+1}}^{\prime}\right|<\varepsilon^{2}$.

Thus, provided that $\varepsilon$ is sufficiently small, the rows of $\bar{A}_{\text {eq } v}$ below the horizontal bar in (4) constitute a Gale transform of a polytope combinatorially equivalent to $Q$.

In analogy to the cubical case, we write $P_{2 r}(Q ; m)$ for the deformed product of $m$-gons with respect to the polytope $Q$ with ordered vertices.

Definition 4.3 The proof of Theorem 4.1 yields a family of projected products of polygons (PDPPs) as the image $\operatorname{PDPP}_{2 r, d}(Q ; m):=\pi_{d}\left(P_{2 r}(Q ; m)\right)$.

En route to a facial description of $\operatorname{PDPP}_{2 r, d}(Q ; m)$, let us pause to introduce a convenient notation for handling products of even polygons combinatorially that bears certain similarities with that of $2 r$-cubes, i.e., products of quadrilaterals. For the even 
polygons above, we label the edge with outer normal $a_{i}$ by $(i, *)$ if $i$ is even and by $(*, i)$ otherwise:

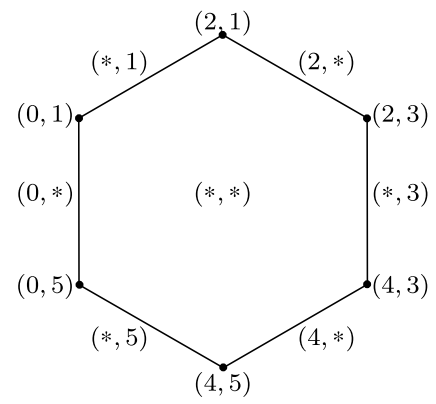

Every vertex is incident to an even edge $(2 i, *)$ and an odd edge $(*, 2 i \pm 1)$ and is labeled by $(2 i, 2 i \pm 1)$. Finally, the polygon itself gets the label $(*, *)$ as the intersection of no edges.

Summing up, the nonempty faces of an even $m$-gon are given by

$$
\begin{aligned}
\mathcal{P}_{m} & =\left\{(2 i, *): 0 \leq i<\frac{m}{2}\right\} & \text { (even edges) } \\
& \cup\left\{(*, 2 i+1): 0 \leq i<\frac{m}{2}\right\} & \text { (odd edges) } \\
& \cup\left\{(2 i, 2 i \pm 1): 0 \leq i<\frac{m}{2}\right\} & \text { (vertices) } \\
& \cup\{(*, *)\} & \text { (polygon) }
\end{aligned}
$$

with inclusion given by the order relation induced by $i \prec *$ for $i \in\{0, \ldots, m-1\}$.

Admittedly, this is neither the most natural nor the most efficient way to encode a polygon combinatorially. However, the following remarks make up for this unusual description. Similar to the description of $2 r$-cubes, the dimension of a face $\left(\alpha_{0}, \alpha_{1}\right) \in \mathcal{P}_{m}$ is the number of $*$-entries. This carries over to products of $m$-gons, i.e., there is an order-preserving bijection between the nonempty faces of an $r$-fold product of $m$-gons and the $r$-fold direct product $\left(\mathcal{P}_{m}\right)^{r}$ with rank function $\operatorname{dim} \alpha=\#\left\{i: \alpha_{i}=*\right\}$ for $\alpha \in\left(\mathcal{P}_{m}\right)^{r}$. Notably most of the results (and proofs) from Sect. 3 carry over to this setting, with only minor modifications.

The key to obtaining a combinatorial description of $\operatorname{PDPP}_{2 r, d}(Q ; m)$ is that, for a vertex $v$ of $P_{2 r}(Q ; m)$, the matrix (4) again encodes a lexicographic triangulation of $Q$. In order to reduce this to the case of neighborly cubical polytopes, after a 
suitable change of basis, the matrix $\bar{A}_{\text {eq } v}$ is of the form

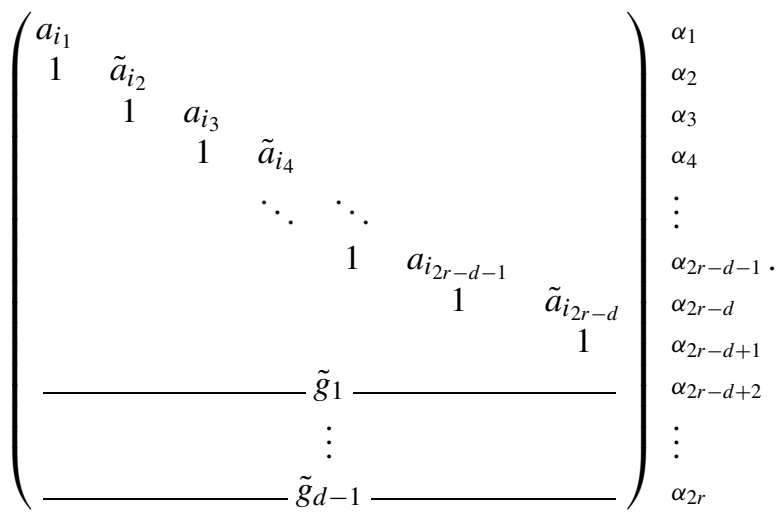

The entries above the diagonal of ones remain to be of order $\varepsilon$. To determine the signs of the entries, which will determine the lexicographic triangulation, let us investigate the local change of the matrix under the change of basis.

In the above combinatorial model for even $m$-gons, the vertex $v$ is identified with a vector $\alpha=\left(\alpha_{1}, \alpha_{2} ; \alpha_{3}, \ldots ; \alpha_{2 r-1}, \alpha_{2 r}\right) \in\left(\mathcal{P}_{m}\right)^{r}$, which corresponds to eq $v$ as indicated. The following table, which is easily established given the coordinates of the normals, summarizes the possible sign patterns in terms of $\alpha$ :

$$
\begin{array}{c||c|c|c|c}
\left(\alpha_{i}, \alpha_{i+1}\right) & (0,1) & (0, m-1) & (2 k, 2 k-1) & (2 k, 2 k+1) \\
\hline\left(\begin{array}{cc}
a_{i} & \tilde{a}_{i+1}
\end{array}\right) & \left(\begin{array}{cc}
-\varepsilon \\
1 & +\varepsilon
\end{array}\right) & \left(\begin{array}{cc}
-\varepsilon & \\
1 & -\varepsilon
\end{array}\right) & \left(\begin{array}{cc}
+\varepsilon & 2 \varepsilon \\
1 & +\frac{2 \varepsilon}{m-2}
\end{array}\right) & \left(\begin{array}{cc}
+\varepsilon & 2 \varepsilon \\
1 & -\frac{2 \varepsilon}{m-2}
\end{array}\right) \\
\hline\left(\sigma_{i}, \sigma_{i+1}\right) & (-,+) & (-,-) & (+,+) & (+,-)
\end{array}
$$

We use the last row, which gathers sign patterns from the diagonal, to define the map

$$
\Phi:\left\{\left(\alpha_{1}, \alpha_{2}\right) \in \mathcal{P}_{m}: \alpha \text { vertex }\right\} \rightarrow\{+,-, 0\}^{2}
$$

with $\Phi\left(\alpha_{1}, \alpha_{2}\right):=\left(\sigma_{1}, \sigma_{2}\right)$ according to the table. Since the face lattice of a convex polytope is atomic, it is easy to see from the definition that $\Phi: \mathcal{P}_{m} \rightarrow\{+,-, 0\}^{2}$ extends to an order- and rank-preserving map from the face poset of an even $m$-gon to that of a 2-cube. The map can be thought of as a folding map:

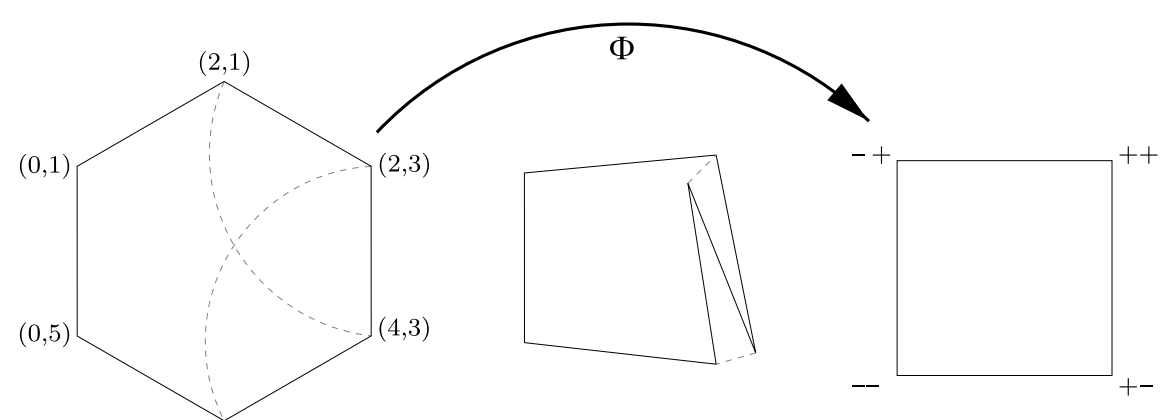

$(4,5)$ 
The induced map $\Phi:\left(\mathcal{P}_{m}\right)^{r} \rightarrow\{+,-, 0\}^{2 r}$ maps faces of $P_{2 r}(k ; m)$ that are strictly preserved under $\pi_{d}$ to surviving faces of $C_{2 r}(Q)$. Phrased differently, the following diagram commutes on the level of faces:

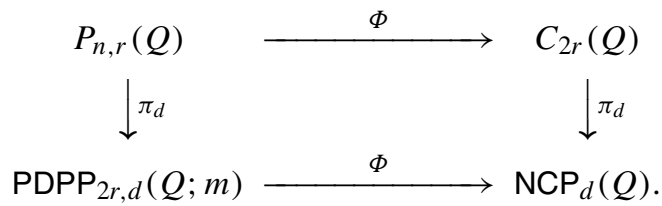

Proposition 4.4 Let $n=2 r$, and let $P=P_{n}(Q ; m)$ and $C=C_{n}(Q)$ be the deformed cube and the product of $m$-gons of dimension $n=2 r$ with respect to $a$ neighborly simplicial $(d-2)$-polytope $Q$ on $n-1$ ordered vertices. Let $v \in P$ be a vertex with eq $v$ represented by $\alpha \in\left(\mathcal{P}_{m}\right)^{r}$, and let $u \in C$ be the vertex corresponding to $\Phi(\alpha) \in\{+,-\}^{n}$. Then $\Phi$ induces an isomorphism of the vertex figures $\operatorname{PDPP}_{n, d}(Q ; m) / \pi_{d}(v)$ and $\operatorname{NCP}_{n}(Q) / \pi_{d}(u)$.

Proof As consistent with the main theme in this article, consider the first $n-d=$ $2 r-d$ coordinates of the inequalities from both $P$ and $C$ that are tight at $v$ and $u$, respectively.

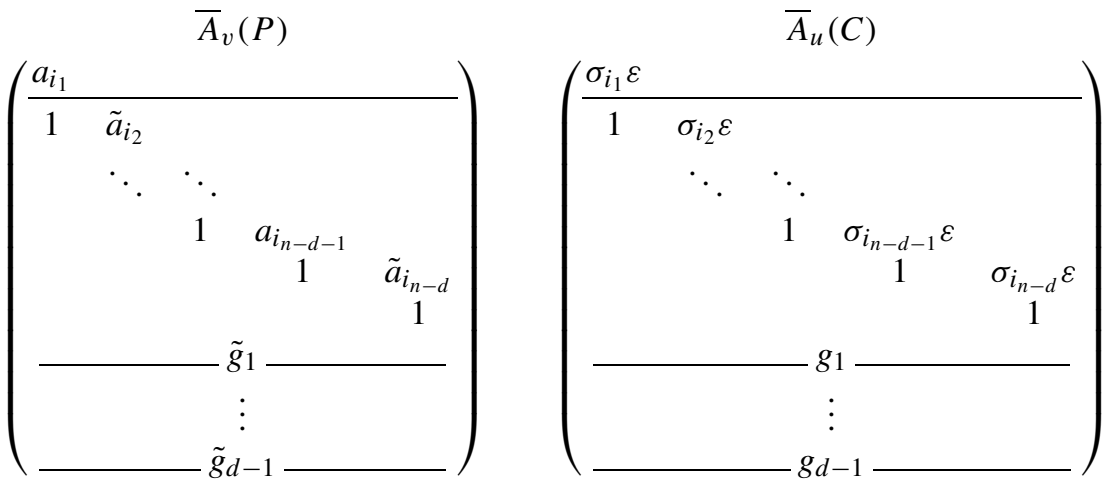

In both matrices, the entries on the secondary diagonal are arbitrary small, and the map $\Phi$ assures that corresponding entries have equal sign. By Lemma 3.5, both $\bar{A}_{v}(P)$ and $\bar{A}_{u}(C)$ are Gale transforms that encode the same lexicographic pyramid $\mathcal{L}_{k}(Q)$. The result now follows by observing that a face $\beta \succeq \alpha$ of $P$ is strictly preserved if and only if $|\beta|$ is a coface of $\mathcal{L}_{k}(Q)$ and $|\Phi(\beta)|=|\beta|$.

This proposition makes way for the combinatorics of the projected deformed products associated with arbitrary neighborly simplicial polytopes.

Theorem 4.5 (Combinatorial description of the PDPPs) Let $P=P_{2 r}(Q ; m)$ be a deformed product of $m$-gons with respect to $Q$, and let $v \in P$ be an arbitrary vertex with eq $v=\alpha \in\left(\mathcal{P}_{m}\right)^{r}$. Then the vertex figure of $\pi_{d}(v)$ in $\operatorname{PDPP}_{2 r, d}(Q ; m)$ is isomorphic to $\mathcal{L}_{p}(Q)$ for

$$
p=\min \left\{i \in[2 r]: \Phi(\alpha)_{i}=-\right\} \cup\{2 r-d-1\} .
$$


In particular, the $(d-1)$-faces of $P$ containing $v$ that are preserved by projection are in one-to-one correspondence to the facets of $\mathcal{L}_{p}(Q)$.

As for the neighborly cubical polytopes, via Shemer's work [15] this result implies a great richness of combinatorial types for the projected products of polygons. In the special case where $Q$ is a cyclic polytope with vertices in standard order, we get a very explicit Gale's evenness-type criterion for the projected products of polygons.

Corollary 4.6 (Combinatorial description of the standard PDPPs) Let $F \subset P=$ $P_{2 r}(Q ; m)$ be a $(d-1)$-face with $Q=\mathrm{Cyc}_{d-2}(2 r-1)$, and let $\beta \in\left(\mathcal{P}_{m}\right)^{r}$ correspond to eq $F$. Then $F$ projects to a facet of $\operatorname{PDPP}_{2 r, d}(Q ; m)$ if and only if $\Phi(\beta)$ satisfies the cubical Gale's evenness criterion.

Acknowledgements The first author would like to thank Andreas Paffenholz, Thilo Rörig, Jakob Uszkoreit, Arnold Waßmer, and Axel Werner for "actively listening" and Vanessa Kääb for more. Both authors gratefully acknowledge support by the German Science Foundation DFG via the Research Training Group "Methods for Discrete Structures" and a Leibniz grant.

\section{References}

1. Amenta, N., Ziegler, G.M.: Deformed products and maximal shadows of polytopes. In: Chazelle, B., Goodman, J., Pollack, R. (eds.) Advances in Discrete and Computational Geometry. Contemporary Mathematics, vol. 223, pp. 57-90. Am. Math. Soc., Providence (1998)

2. Babson, E.K., Billera, L.J., Chan, C.S.: Neighborly cubical spheres and a cubical lower bound conjecture. Israel J. Math. 102, 297-315 (1997)

3. De Loera, J.A., Rambau, J., Santos, F.: Triangulations: Applications, Structures, Algorithms. Book in preparation

4. Goldfarb, D.: Worst case complexity of the shadow vertex simplex algorithm. Technical report, Department of Industrial Engineering and Operations Research, Columbia University, USA (1983)

5. Grünbaum, B.: Convex Polytopes, 2nd edn. Graduate Texts in Mathematics, vol. 221. Springer, New York (2003). Kaibel, V., Klee, V., Ziegler, G.M. (eds. of 2nd edn.). Original edition: Interscience, London (1967)

6. Joswig, M., Rörig, T.: Neighborly cubical polytopes and spheres. Israel J. Math. 159, 221-242 (2007)

7. Joswig, M., Ziegler, G.M.: Neighborly cubical polytopes. Discrete Comput. Geom. 24, 325-344 (2000)

8. Klee, V., Minty, G.J.: How good is the simplex algorithm? In: Shisha, O. (ed.) Inequalities, vol. III, pp. 159-175. Academic Press, New York (1972)

9. Lee, C.W.: Regular triangulations of convex polytopes. In: Gritzmann, P., Sturmfels, B. (eds.) Applied Geometry and Discrete Mathematics: The Victor Klee Festschrift, pp. 443-456. Am. Math. Soc., Providence (1991)

10. McMullen, P., Schulz, C., Wills, J.M.: Polyhedral 2-manifolds in $E^{3}$ with unusually large genus. Israel J. Math. 46, 127-144 (1983)

11. Rörig, T., Sanyal, R.: Non-projectability of polytope skeleta. Preprint in preparation (2007)

12. Rörig, T., Ziegler, G.M.: Polyhedral surfaces in wedge products. Preprint in preparation (2007)

13. Sanyal, R.: On the combinatorics of projected deformed products. Diplomarbeit, TU Berlin, 57 pages. http://www.math.tu-berlin.de/ sanyal/diploma/DiplomaThesis.pdf (2005)

14. Sanyal, R.: Topological obstructions for vertex numbers of Minkowski sums. J. Comb. Theory Ser. A 116, 168-179 (2009)

15. Shemer, I.: Neighborly polytopes. Israel J. Math. 43, 291-314 (1982)

16. Ziegler, G.M.: Lectures on Polytopes. Graduate Texts in Mathematics, vol. 152. Springer, New York (1995). Revised 7th edn. (2007) 
17. Ziegler, G.M.: Face numbers of 4-polytopes and 3-spheres. In: Tatsien, L. (ed.) Proceedings of the International Congress of Mathematicians (ICM 2002, Beijing), vol. III, pp. 625-634. Higher Education Press, Beijing (2002)

18. Ziegler, G.M.: Projected products of polygons. Electron. Res. Announc. Am. Math. Soc. 10, 122-134 (2004). http://www.ams.org/era/2004-10-14/S1079-6762-04-00137-4

19. Ziegler, G.M.: Projected polytopes, Gale diagrams, and polyhedral surfaces. Oberwolfach reports, No 2, pp. 986-989 (2005) 